\title{
Formation of a Fast Charge Transfer Channel in Quasi-2D Perovskite Solar Cells through External Electric Field Modulation
}

\author{
Xixiang Zhu ${ }^{1,3}$, Liping Peng ${ }^{2}$, Jinpeng $\mathrm{Li}^{1}{ }^{1}$, Haomiao $\mathrm{Yu}^{1}$ and Yulin $\mathrm{Xie}^{2, *(\mathbb{D})}$ \\ 1 Key Laboratory of Luminescence and Optical Information, Department of Physics, School of Science, Beijing \\ Jiaotong University, Ministry of Education, Beijing 100044, China; xxzhu@bjtu.edu.cn (X.Z.); \\ jinpengli@bjtu.edu.cn (J.L.); yuhaomiao@bjtu.edu.cn (H.Y.) \\ 2 School of Physics and Electronics, Huanggang Normal University, Huanggang 438000, China; \\ pengliping@hgnu.edu.cn \\ 3 School of Electronic and Information Engineering, Beijing Jiaotong University, Beijing 100044, China \\ * Correspondence: xieyulin@hgnu.edu.cn
}

Citation: Zhu, X.; Peng, L.; Li, J.; Yu, H.; Xie, Y. Formation of a Fast Charge Transfer Channel in Quasi-2D Perovskite Solar Cells through External Electric Field Modulation. Energies 2021, 14, 7402. https:// doi.org/10.3390/en14217402

Academic Editor: Antonino S. Aricò

Received: 30 September 2021

Accepted: 3 November 2021

Published: 5 November 2021

Publisher's Note: MDPI stays neutral with regard to jurisdictional claims in published maps and institutional affiliations.

Copyright: (c) 2021 by the authors. Licensee MDPI, Basel, Switzerland. This article is an open access article distributed under the terms and conditions of the Creative Commons Attribution (CC BY) license (https:// creativecommons.org/licenses/by/ $4.0 /)$.

\begin{abstract}
Quasi-2D perovskites solar cells exhibit excellent environmental stability, but relatively low photovoltaic properties, compared with 3D perovskites solar cells. However, charge transport and extraction in quasi-2D perovskite solar cells are still limited by the inevitable quantum well effect, resulting in low power conversion efficiency (PCE). To date, most efforts concentrate on crystal orientation and favorable alignment during materials and films processing. In this paper, we demonstrated that the quasi-2D perovskite $\left[(\mathrm{BA})_{2}(\mathrm{MA})_{3} \mathrm{~Pb}_{4} \mathrm{I}_{13}(n=4)\right]$ solar cells show an optimized device performance through forming a fast charge transfer channel among 2D quantum wells through external electric field modulation, with appropriate modulation bias and time after the device has been fabricated. Essentially, ions will move directionally due to local polarization in quasi-2D perovskite solar cells under the action of electric field modulation. More importantly, the mobile ions function as a dopant to de-passivate the defects when releasing at grain boundaries, while decreasing built-in potential by applying forward modulation bias with proper modulation time. The capacitance-voltage characteristics indicate that electric field modulation can decrease the charge accumulation and improve the charge collection in quasi-2D perovskite solar cells. Photoluminescence (PL) studies confirm that the non-radiative recombination is reduced by electric field modulation, leading to enhanced charge transfer. Our work indicates that external electric field modulation is an effective method to form a fast charge transfer channel among 2D quantum wells, leading to enhanced charge transfer and charge collection through local polarization toward developing high-performance quasi-2D perovskite devices.
\end{abstract}

Keywords: quasi-2D perovskite; ions; electric field modulation

\section{Introduction}

Quasi-2D perovskite solar cells have shown great potential in future photovoltaic applications, due to their tunable energy level and comparable stability [1-7]. Basically, quasi2D perovskites are prepared with the Ruddlesden-Popper perovskite (RPP) nanoplates structures, which has been an effective method for enhancing the stability for photovoltaic applications [8-10]. Due to the disconnected inorganic semiconducting network in the direction of the spacing cations in the RPP structure, the charge carrier transport is drastically limited, decreasing the photocurrent in quasi-2D perovskites solar cells [11,12]. Recent studies have introduced 2D perovskite nanoplates processing methods, such as hot-casting or vacuum fabrication method, to orient $2 \mathrm{D}$ perovskites preferentially in the out-of-plane direction, where the 2D nanoplates can efficiently connect the electron and hole selective electrode layers with improved charge carrier transport and device performance [12-14]. Furthermore, the layered structure of low-dimensional perovskite can effectively inhibit ion migration, thereby reducing the degradation of perovskite caused by ion diffusion 
or the corrosion in charge transport, which is beneficial to improve the stability of perovskite [15-17]. Nevertheless, ion migration still exists in such quasi-2D perovskites. It becomes the main reason that causes the device to have a hysteresis effect, giving rise to the impact on charge carrier transfer or energy transfer between 2D quantum wells [18-20]. It should be noted that the hysteresis effect caused by exogenous ions may even be more substantial than intrinsic ions [21,22]. Moreover, electric field modulation is a crucial method to explore the migration of perovskite ions [23-25]. Recently, PL studies indicated that charge-transfer excitons can be formed at the interface within 2D perovskite heterostructures to realize a broad light emission [26]. Therefore, exploring the external electric field modulation effect on the detailed charge carrier dynamics and the corresponding RPPs structural correlation of quasi-2D perovskites involved in internal photovoltaic processes remains valuable, and is essential to achieve more useful photogenerated carriers.

To date, a lot of work reported the performance of 2D perovskite solar cells could be improved through film or materials processing. However, it is rarely reported on the use of methods to further improve device performance after the device has been fabricated. In this work, we develop a simple and effective method to enhance the charge transfer among 2D quantum wells in quasi-2D perovskite solar cells, with the aim of further improving the PCE after the device has been fabricated. A quasi-2D perovskite $\left[(\mathrm{BA})_{2}(\mathrm{MA})_{3} \mathrm{~Pb}_{4} \mathrm{I}_{13}(n=4)\right]$ solar cell was prepared by changing the preheating temperature, and annealing temperature of the perovskite layer. We should note that even the enhanced charge transport/extraction and final power conversion efficiency (PCE) in $\mathrm{BA}_{2} \mathrm{MA}_{3} \mathrm{~Pb}_{4} \mathrm{I}_{13}$ based quasi-2D perovskite solar cells benefit from efforts to change the vertical arrangement of the BA spacer cations, and the impact of quantum wells is still inevitable, and even under optimal conditions, it will cause the electrical field-dependent charge extraction and fill factor (FF) reduction in the quasi-2D perovskite solar cell [27]. Here, we found that a fast charge transfer channel can be formed among 2D quantum wells by applying external electric field modulation with forwarding bias at a constant time through local electric polarization in quasi-2D perovskite solar cells. By combining capacitance-voltage $(C-V)$ and PL characteristics studies, we demonstrate that fast charge transfer can be ascribed to the enhanced builtin potential due to passivation of defects at grain boundaries, while decreasing built-in potential by applying forward bias through enhanced local polarization. Importantly, revealing the effects of electric field modulation on the charge transport/collection and recombination provides a constructive scheme of quasi-2D perovskites solar cells toward further promoting photovoltaic properties.

\section{Materials and Methods}

\subsection{Material Processing and Device Fabrication}

The quasi-2D solar cells were prepared in the p-i-n structure of the ITO/PEDOT: $\mathrm{PSS} /(\mathrm{BA})_{2} \mathrm{MA}_{3} \mathrm{~Pb}_{4} \mathrm{I}_{13} / \mathrm{PCBM} / \mathrm{PEI} / \mathrm{Ag}$ preheating method to explore the effects of electric field modulation on device performance. The perovskite precursor comprised $\mathrm{PbI}_{2}$ (99.999\%, Sigma-Aldrich, St. Louis, MO, USA), MAI (99\%, Shanghai Materwin, Shanghai, China), and BAI (99.5\%, Xi'an Polymer, Xi'an, China), with a 4:3:2 molar ratio in a mixed solvent of $\mathrm{N}, \mathrm{N}-$ dimethylformamide (DMF, 99.9\%, Alfa Aesar, Ward Hill, MA, USA) and dimethyl sulfoxide (DMSO, 99.9\%, Sigma-Aldrich) in 9:1 volume with a concentration of $0.8 \mathrm{M}$. Before coating, the indium tin oxides glass (ITO) glass substrates were cleaned and then exposed to plasma for $5 \mathrm{~min}$. The hole transporting layer poly $(3,4-$ ethylenedioxythiophene) poly(styrenesulfonate) (PEDOT:PSS) 4083 (Xi'an Polymer) was coated on ITO at $4000 \mathrm{rpm}$ for $40 \mathrm{~s}$, and then dried in an oven at $120^{\circ} \mathrm{C}$ for $30 \mathrm{~min}$. Before covering the perovskite film, the ITO/ PEDOT:PSS substrates were preheated on a hot plate at $100{ }^{\circ} \mathrm{C}$ for $3-5 \mathrm{~min}$. Then, the regulated perovskite solution was deposited by dropping $40 \mu \mathrm{L}$ on the ITO/ PEDOT:PSS substrates at $5000 \mathrm{rpm}$ for $30 \mathrm{~s}$. The as-coated sample was dried at $100{ }^{\circ} \mathrm{C}$ for $10 \mathrm{~min}$. The electron transport layer PCBM (Lumtech, 99.5\%, Moorestown, NJ, USA) was dissolved in chlorobenzene (Sigma-Aldrich, 99.8\%), and coated on the perovskite film at $2000 \mathrm{rpm}$ for $45 \mathrm{~s}$ at a concentration of $20 \mathrm{mg} / \mathrm{mL}$. The 
PEI (0.1 wt \% in isopropanol) was spin-coated at $5000 \mathrm{rpm}$ for $50 \mathrm{~s}$. Finally, a $100 \mathrm{~nm}$ silver electrode was evaporated on the substrates under a pressure of $3 \times 10^{-4} \mathrm{~Pa}$, and the active area of all devices was $0.055 \mathrm{~cm}^{2}$.

\subsection{Device Characterization}

The device performances ( $J-V$ curves) were obtained using a Newport Thermal Oriel 96000 with Keithley 2400 under the illumination of 1 sun with a $20 \mathrm{mV} \mathrm{s}^{-1}$ scan rate in air. The morphology of quasi-2D perovskite films were observed with a Bruker Dimension 3100 and Quanta 3D FEG-FIB. The luminescence spectrum was recorded with an Edinburgh FLS920. A HORIBA Scientific DeltaPro was used to record the PL of the quasi-2D perovskite. An Agilent 4294A impedance spectroscopy analyzer was used to record $C-V$ spectra at a dark state.

\section{Results}

\subsection{Device Section}

The $J-V$ characteristic curves of quasi-2D solar cells are shown in Figure 1a, with different preheating temperatures under AM 1.5 G are measured using forward and reversed scan, and the corresponding photovoltaic parameters are shown in Table 1. Among them, $0 \mathrm{~V}$ bias is the original device without any external electric field.
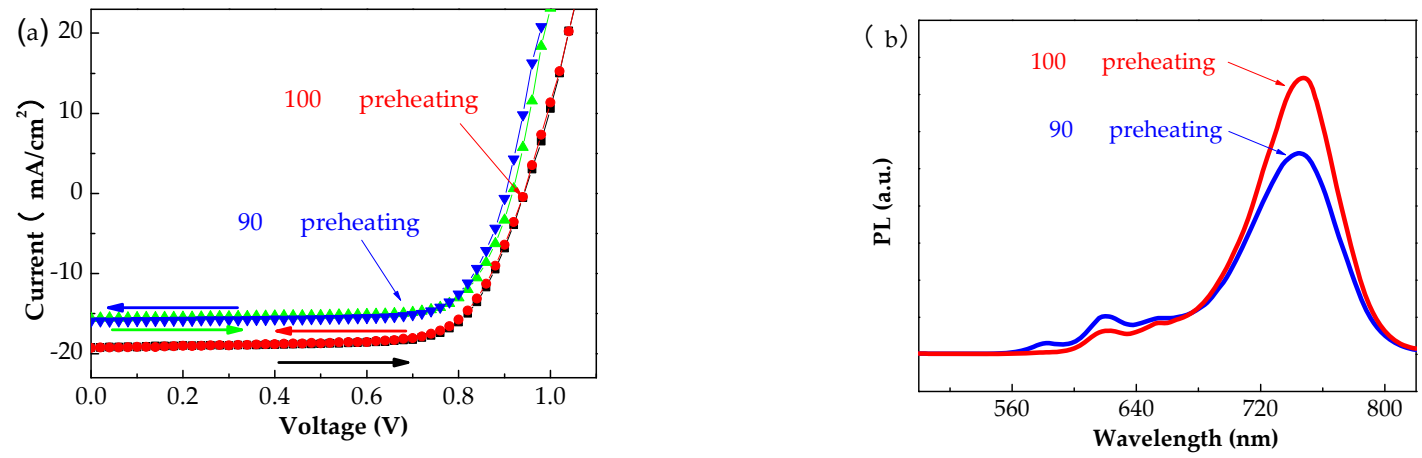

Figure 1. (a) $J-V$ curve of quasi-2D perovskite $\left[(\mathrm{BA})_{2} \mathrm{MA}_{3} \mathrm{~Pb}_{4} \mathrm{I}_{13}, n=4\right]$ solar cells processing with preheating of $90{ }^{\circ} \mathrm{C}$ and $100{ }^{\circ} \mathrm{C}$ under forward and reversed scan. (b) PL spectra of perovskite films from front sides at $90{ }^{\circ} \mathrm{C}$ and $100{ }^{\circ} \mathrm{C}$ preheating temperatures.

Table 1. Photovoltaic parameters of electric field modulated quasi-2D perovskite solar cells under different modulation bias at $200 \mathrm{~s}$ with the same batch of devices.

\begin{tabular}{ccccc}
\hline Modulation Bias & $\boldsymbol{V}_{\mathbf{O C}}(\mathbf{V})$ & $J_{\text {SC }}\left(\mathbf{m A} \cdot \mathbf{c m}^{-\mathbf{2}}\right)$ & FF (\%) & PCE (\%) \\
\hline $0 \mathrm{~V}$ & 1.03 & 20.14 & 63.89 & 13.2 \\
$0.8 \mathrm{~V}$ & 1.04 & 20.28 & 63.69 & 13.45 \\
$1.0 \mathrm{~V}$ & 1.04 & 20.06 & 63.57 & 13.27 \\
$1.5 \mathrm{~V}$ & 1.10 & 19.22 & 59.03 & 12.48 \\
$-0.1 \mathrm{~V}$ & 1.10 & 18.11 & 63.00 & 12.55 \\
$-0.5 \mathrm{~V}$ & 0.87 & 18.48 & 32.97 & 5.27 \\
\hline
\end{tabular}

Clearly, the photocurrent hysteresis of all devices can be ignored. It can be seen that the higher efficiency of $13.2 \%\left(V_{\mathrm{OC}}=0.94 \mathrm{~V}, J_{\mathrm{SC}}=19.24 \mathrm{~mA} \cdot \mathrm{cm}^{-2}, \mathrm{FF}=0.73\right)$ is obtained when using $100{ }^{\circ} \mathrm{C}$ to preheat the PEDOT:PSS samples, as compared with the devices without $90{ }^{\circ} \mathrm{C}$ preheating $\mathrm{PCE}=10.8 \%\left(V_{\mathrm{OC}}=0.92 \mathrm{~V}, J_{\mathrm{SC}}=15.6 \mathrm{~mA} \cdot \mathrm{cm}^{-2}, \mathrm{FF}=0.75\right)$. We also note that when the PL spectra of quasi-2D perovskites is under the preheating condition of $100{ }^{\circ} \mathrm{C}$, the main peak of PL intensity of the quasi-2D perovskite film is greater than that of the quasi-2D perovskite film under the preheating condition of $90{ }^{\circ} \mathrm{C}$ (Figure $1 \mathrm{~b}$ ). This indicates that increasing the preheating temperature can promote the radiative recombination luminescence of the quasi-2D perovskite (Figure S1). The corresponding mobility of 
the device is $6.3 \times 10^{-3} \mathrm{~cm}^{2} \cdot \mathrm{V}^{-1} \cdot \mathrm{s}^{-1}$ under the illumination of $0.54 \mathrm{~mW} \cdot \mathrm{cm}^{-2}$, measured from the charge extraction by linear increasing voltage (CELIV) method (Figure S2).

\subsection{AFM Methodology}

Morphology is an important parameter that affects device performance. Figure 2a shows the morphology of $2 \mathrm{D}$ perovskite films; when preheating at $90{ }^{\circ} \mathrm{C}$, the surface morphology of perovskite shows partial cracks and small grain size, indicating that there are many defects on the surface of quasi-2D perovskites prepared under preheating at $90{ }^{\circ} \mathrm{C}$ and uneven film formation, which will reduce the photovoltaic efficiency of $2 \mathrm{D}$ perovskite solar cells. When the preheating temperature is increased to $100{ }^{\circ} \mathrm{C}$, the perovskite crystallizes well, and the grain size becomes larger and compact (Figure 2a,b). The better crystallization of perovskite can inhibit migration and enhance charge transmission and collection.

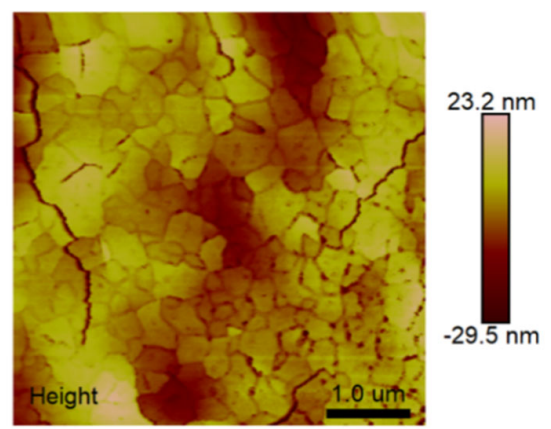

(a)

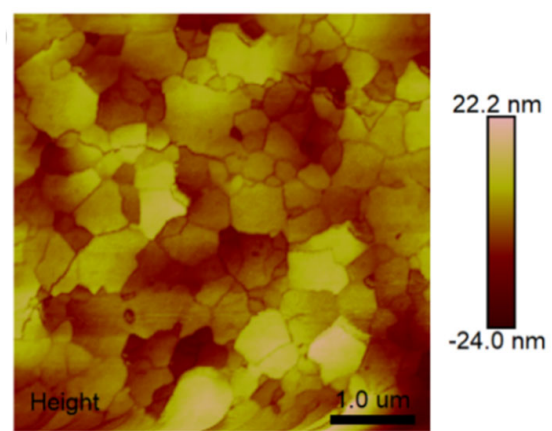

(b)

Figure 2. Surface morphology of $(\mathrm{BA})_{2} \mathrm{MA}_{3} \mathrm{~Pb}_{4} \mathrm{I}_{13}$ with (a) preheating at $90{ }^{\circ} \mathrm{C}$ and (b) preheating at $100{ }^{\circ} \mathrm{C}$.

\subsection{Signal Analysis (Electric Field Modulation and Impedance Spectra Analysis)}

Now, we discuss the charge transfer of quasi-2D perovskite solar cells by electric field modulation. It is known that, under the action of the external electric field, ions will move in a directional way, which affects the charge transfer process of perovskite devices. Essentially, using proper external electric field modulation presents a new approach to improve the charge transfer between quantum wells and quasi-2D perovskite solar cells, as schematically illustrated in Figure 3. Here, the photovoltaic performance of quasi-2D perovskites is monitored by applying forward or reversed bias with different modulation times.

The $J-V$ curve under applied other modulation times is shown in Figure 4a, a forward bias at a constant time of $200 \mathrm{~s}$. When $+0.8 \mathrm{~V}$ bias was applied, the devices yielded a high PCE of $13.45 \%$, deriving from a $J_{\mathrm{SC}}$ of $20.28 \mathrm{~mA} \mathrm{~cm}{ }^{2}$, an FF of $63.69 \%$, and a $V_{\mathrm{OC}}$ of $1.04 \mathrm{~V}$. The photovoltaic parameters of quasi-2D perovskite devices were gradually reduced, along with the applied forward bias increment, indicating that ions movement would affect the charge transfer process of quasi-2D perovskite solar cells. The photovoltaic parameters are improved when the used forward bias $V_{\text {app }}$ is less than the built-in potential $\left(\mathrm{V}_{\mathrm{bi}}\right)$; when $\mathrm{V}_{\mathrm{app}}>\mathrm{V}_{\mathrm{bi}}, V_{\mathrm{OC}}$ increases, $J_{\mathrm{SC}}$ and PCE decreases. Essentially, the quasi-2D perovskite solar cells can generate a built-in electric field to cause the directional movement of charge, and facilitate the separation of charge carriers. The shielding electric field will enhance the local polarization in quasi-2D perovskite solar cells, leading to the directional movement of ions under the action of the external electric field. Due to the shielding effect of the electric field, the charge carrier diffusion at the interface will weaken or even disappear, and the shielding electric field generated will also disappear. However, suppose that the forward bias is too large, and the charge carrier begins to be injected. In that case, the effect of shielding electric fields will be weakened, leading to a decrease in device performance [28]. Interestingly, the efficiency of quasi-2D perovskites solar cells 
are decreased by applying reversed bias, as shown in Figure $4 \mathrm{~b}$. It can be seen that when the reversed bias voltage of $-0.1 \mathrm{~V}$ is applied, $V_{\mathrm{OC}}$ increases, but the device performance decreases. Moreover, $V_{\mathrm{OC}}$ and FF decrease, obviously, and the PCE significantly reduced by decreasing the reversed bias to $-0.5 \mathrm{~V}$. When the reversed bias is applied, $\mathrm{I}^{-}$moves towards the Ag electrode and reacts with it, leading to the enhanced ion migration and destruction of the region corresponding to the reversed bias electrode, weakening the device performance [29]. The corresponding photovoltaic parameters under forwarding or reversed bias at a constant time are summarized in Table 1. In the same modulation voltage of $2 \mathrm{~V}$, the effect of different modulation times on device performance is shown in Figure $4 \mathrm{c}$. The corresponding photovoltaic parameters are shown in Table 2.
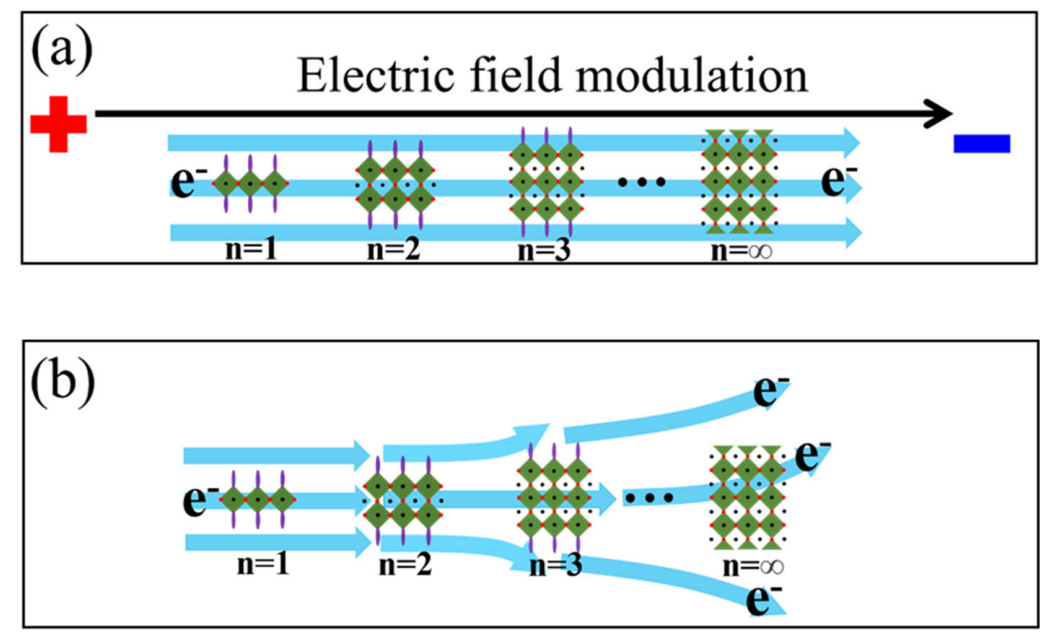

Figure 3. Schematic of (a) fast charge transfer channel between quantum wells through external electric field modulation in quasi-2D perovskite solar cells. (b) Inefficient random charge transfer in quasi-2D perovskite solar cells.
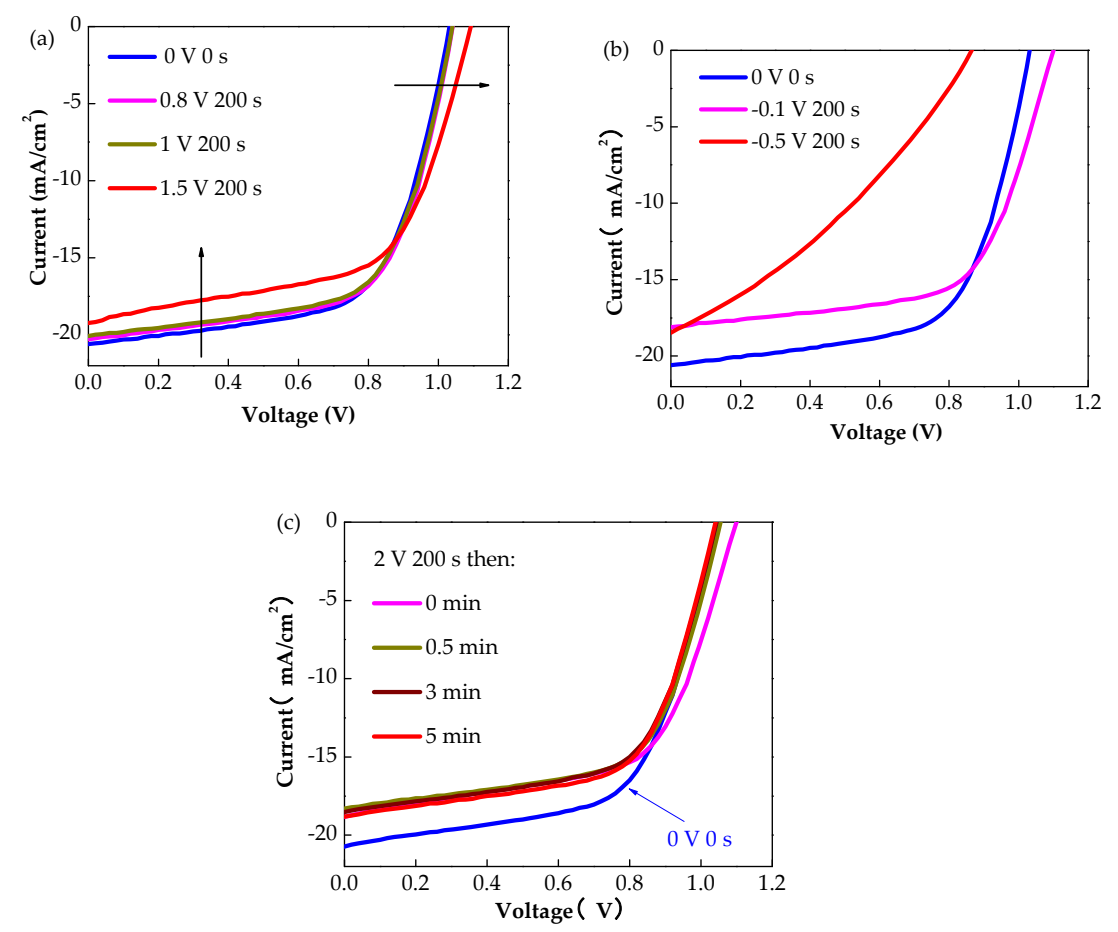

Figure 4. $J-V$ curve of quasi-2D perovskite $\left[(\mathrm{BA})_{2} \mathrm{MA}_{3} \mathrm{~Pb}_{4} \mathrm{I}_{13}, n=4\right]$ solar cells under external electric field modulation with (a) different forward bias at constant time of $200 \mathrm{~s}$. (b) Other reversed bias at a continuous time of $200 \mathrm{~s}$. (c) Other modulation times at consistent forward bias of $2 \mathrm{~V}$. 
Table 2. Photovoltaic parameters of electric field-modulated quasi-2D perovskite solar cells under different modulation times, at a constant forward bias with the same batch of devices.

\begin{tabular}{cccccc}
\hline Modulation Bias & Time (Second) & $V_{\text {OC }}(\mathbf{V})$ & $J_{\text {SC }}\left(\mathbf{m A} \cdot \mathbf{c m}^{-\mathbf{2}}\right)$ & FF (\%) & PCE (\%) \\
\hline $0 \mathrm{~V}$ & $0 \mathrm{~s}$ & 1.03 & 20.14 & 63.89 & 13.20 \\
& $200 \mathrm{~s}$ & 1.10 & 18.82 & 59.89 & 12.40 \\
$2 \mathrm{~V}$ & $230 \mathrm{~s}$ & 1.06 & 18.30 & 62.51 & 12.07 \\
& $380 \mathrm{~s}$ & 1.04 & 18.52 & 62.06 & 12.00 \\
& $500 \mathrm{~s}$ & 1.04 & 18.82 & 62.26 & 12.20 \\
\hline
\end{tabular}

The quasi-2D perovskite solar cells demonstrate a worse performance by adding $2 \mathrm{~V}$ positive bias and a longer modulation time. We should note that the $V_{\mathrm{OC}}$ can quickly be recovered to its initial value, but the efficiency could not be restored. When $2 \mathrm{~V}$ bias modulation is applied again for $20 \mathrm{~s}$, both the $V_{\mathrm{OC}}$ and PCE can be improved (Table 3 ). In addition, after the device is placed in the glove box with a pure nitrogen environment for $24 \mathrm{~h}$, the PCE can be restored to the initial level (Table 3) [30].

Table 3. Photovoltaic parameters of electric field modulated quasi-2D perovskite solar cells placed in the glove box with a pure nitrogen environment after electric field modulation for $1 \mathrm{~min}$ and $24 \mathrm{~h}$ with the same batch of devices.

\begin{tabular}{cccccc}
\hline Modulation Bias & Restore Time & $V_{\text {OC }}(\mathrm{V})$ & $J_{\text {SC }}\left(\mathbf{m A} \cdot \mathbf{c m}^{-2}\right)$ & FF (\%) & PCE (\%) \\
\hline $2 \mathrm{~V}$ & $20 \mathrm{~s}$ & 1.12 & 17.7 & 62.25 & 12.34 \\
0 & $24 \mathrm{~h}$ & 1.06 & 20.37 & 63.77 & 13.75 \\
\hline
\end{tabular}

The effect of external electric field modulation on the internal electric field of the device can be analyzed according to the impedance spectrum. As shown in Figure 5a, the device capacitance peak will shift to the right when applying a forward bias of $1.5 \mathrm{~V}$ for $200 \mathrm{~s}$, and the corresponding capacitance peak will also increase significantly. This indicates that the internal electric field reduced using electric field modulation, leading to the weakening of electric polarization and surface charge, and the increase in total charge, namely, the rise of device capacitance. Figure $5 \mathrm{~b}$ show the $C^{-2}-V$ relationship and the profiles exhibit the typical Mott-Schottky behavior for the quasi-2D perovskite solar cells. The values of $\mathrm{V}_{\mathrm{bi}}$ can be derived via a straight-line fitting to the Mott-Schottky curve by the relationship (1):

$$
C^{-2}=\frac{2}{\mathrm{~A}^{2} q \varepsilon \varepsilon_{0} \mathrm{~N}_{\mathrm{A}}}\left(\mathrm{V}_{\mathrm{bi}} \mathrm{V}_{\mathrm{app}}\right)
$$

where $q$ is the elementary charge $[31,32]$. By linear fitting of $C^{-2}-V$ curve, the $\mathrm{V}_{\mathrm{bi}}$ of the device without and with modulation is $1.0 \mathrm{~V}$ and $1.2 \mathrm{~V}$, respectively. The results are consistent with the $V_{\mathrm{OC}}$ in $J-V$ curve, which further indicates that the forward bias of $0.8 \mathrm{~V}$ for $200 \mathrm{~s}$ can inhibit the ion migration, and enhance the charge transfer of quasi-2D perovskite solar cells.
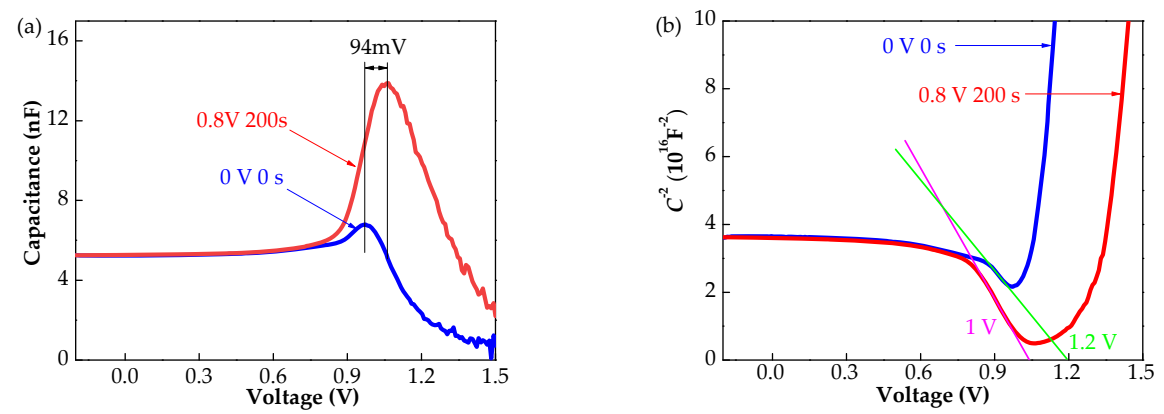

Figure 5. Impedance spectrum of electric field modulated quasi-2D perovskite solar cells (a) The $C-V$ spectra. (b) The corresponding Mott-Schottky $\left(C^{-2}-V\right)$ curves. 


\section{Discussion}

We now discuss the effect of external electric field modulation on charge transfer between quantum wells in quasi-2D perovskite solar cells using the PL method. When the external conditions are changed, such as photoexcitation and electric field modulation, the peak position and intensity of the PL spectrum will change due to ion migration, specifically, because ion migration, $\mathrm{MA}^{+}$, or $\mathrm{I}^{-}$in perovskites will gradually accumulate, resulting in obvious PL quenching. Figure 6a exhibits the PL spectrum of quasi-2D perovskite solar cells with increased forward bias at constant time of $60 \mathrm{~s}$. When $\mathrm{V}_{\text {bias }}=0.8 \mathrm{~V}$, that is, $\mathrm{V}_{\mathrm{bias}}<\mathrm{V}_{\mathrm{bi}}$, the PL emission intensity becomes stronger, indicating that the radiative recombination emission of the quasi-2D perovskite becomes stronger under this condition, and the corresponding non-radiative recombination emission becomes weaker, which can generate more charge carriers. However, when $\mathrm{V}_{\text {bias }}>\mathrm{V}_{\mathrm{bi}}$, the PL emission intensity becomes negligible, indicating that the non-radiative recombination suppresses the generation and transport of charge carriers. Interestingly, the quasi-2D perovskite solar cells can be restored to the original state after being placed in a glove box or dark environment for a period of time after the cancellation of modulation. In order to further explore the role of the external electric field modulation between different- $n$-value nanoplates in the quasi-2D perovskites solar cells, we started with the characterization for the same devices using the $\Delta \mathrm{PL} / \mathrm{PL}_{0}$ method. Here, the $\Delta \mathrm{PL} / \mathrm{PL}_{0}$ is defined by Equation (2):

$$
\Delta \mathrm{PL} / \mathrm{PL}_{0}=\frac{\mathrm{PL}_{1}-\mathrm{PL}_{0}}{\mathrm{PL}_{0}}
$$

where $\mathrm{PL}_{0}$ And $\mathrm{PL}_{1}$ are the PL intensities without and with electric field modulation, respectively. In general, quasi-2D perovskites are normally formed with different- $n$-value nanoplates through crystallization $[33,34]$. The energy transfer between different- $n$-value nanoplates is the key issue determining the optical properties in 2D perovskites. We should also note that energy transfer relies on carrier transport in quasi-2D perovskite films. Essentially, PL quenching can effectively reflect the recombination process in quasi-2D perovskites. Therefore, $\triangle \mathrm{PL} / \mathrm{PL}_{0}$ can be used to study charge transport between different- $n$ value nanoplates in quasi-2D perovskite solar cells under working conditions. Here, when applied bias of $0.8 \mathrm{~V}$, the $\Delta \mathrm{PL} / \mathrm{PL}_{0}$ of small $n$ value become negligible. On the contrary, the $\Delta \mathrm{PL} / \mathrm{PL}_{0}$ of small $n$ value peak become appreciable when the electric field modulation increases to $1.5 \mathrm{~V}$ and $2 \mathrm{~V}$ (Figure $6 \mathrm{~b}$ ). Our negligible and appreciable $\Delta \mathrm{PL} \mathrm{PL}_{0}$ of small $n$ value with and without proper forward bias further supports that electric field modulation can effectively control the charge transfer between different- $n$-value nanoplates. Figure $6 c$ shows the PL spectra of quasi-2D perovskites under a different reversed bias. When the modulation bias is set to $-0.1 \mathrm{~V}$ and $-0.5 \mathrm{~V}$, the PL spectra of devices quench rapidly, indicating that the radiative recombination luminescence of devices becomes weaker, the corresponding non-radiative recombination becomes stronger, and suppress the charge transfer between different- $n$-value nanoplates. Figure $6 \mathrm{~d}$ demonstrates the $\triangle \mathrm{PL} / \mathrm{PL}_{0}$ curve with reversed bias, the negligible $\Delta \mathrm{PL} / \mathrm{PL}_{0}$ of small $n$ value further indicates the PL intensity rapid quenching give rise to inhibits the transport of the charge carrier. The trend of PL studies is consistent with the $J-V$ results of the device under reversed bias.

Moreover, The PL characteristics of the devices with the same modulation bias but different modulation time is also analyzed. Figure 7a shows the steady-state PL spectra of the devices after $0 \mathrm{~min}, 3 \mathrm{~min}, 5 \mathrm{~min}, 7 \mathrm{~min}, 10 \mathrm{~min}$, and $30 \mathrm{~min}$, respectively. With the increase of modulation time, the PL intensity becomes stronger, which indicates the non-radiative recombination of the devices becomes weaker. Furthermore, when reversed modulation bias of $-0.5 \mathrm{~V}$ is applied, the PL intensity increased with modulation time, indicating that, under the reversed bias with the same direction as the $\mathrm{V}_{\mathrm{bi}}$, the charge carrier recombination is enhanced, leading to enhanced radiative recombination (Figure $7 \mathrm{~b}$ ). Clearly, our results indicate that the external electric field modulation is indeed responsible for forming fast charge transfer channel between quantum wells quasi-2D perovskite solar cells. 

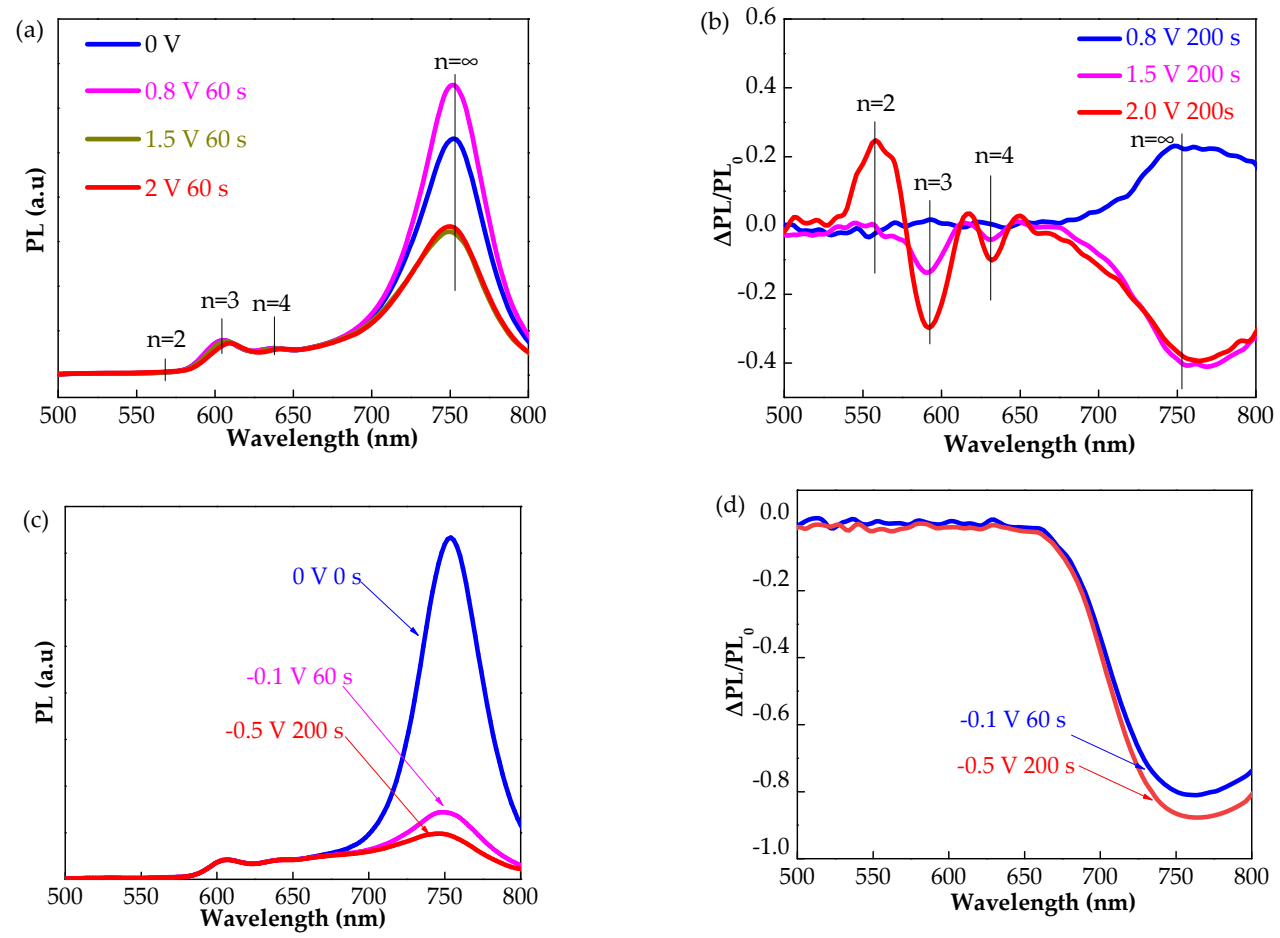

Figure 6. Optical characteristic spectra of electric field modulated quasi-2D perovskite solar cells. Under different, forward bias at constant time: (a) PL spectra. (b) $\Delta \mathrm{PL} / \mathrm{PL}_{0}$ curve. Under other reversed bias with vary time: (c) PL spectra. (d) $\Delta \mathrm{PL} / \mathrm{PL}_{0}$ curve.
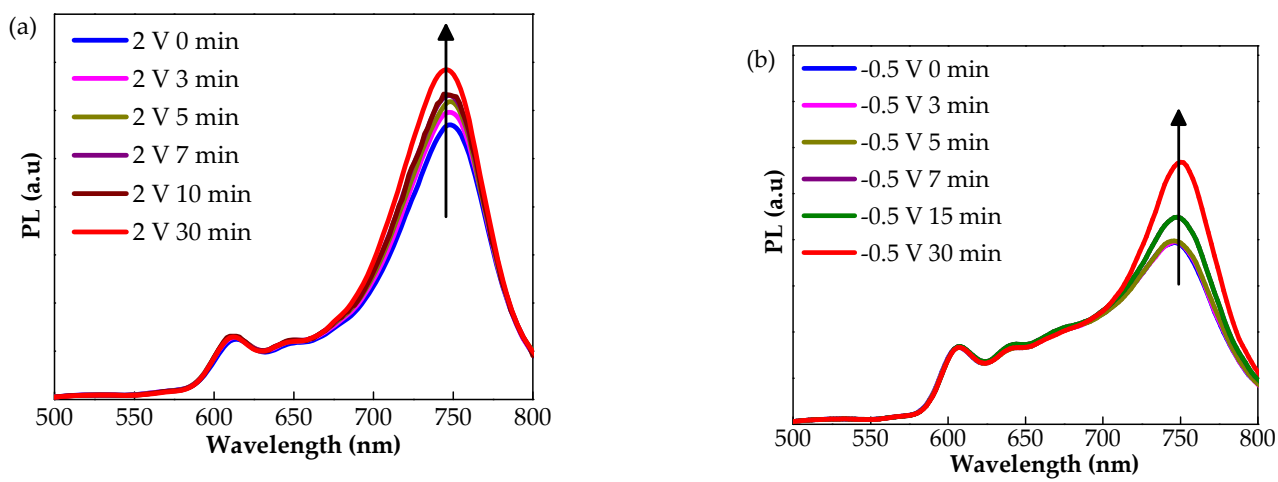

Figure 7. PL spectra of electric field modulated quasi-2D perovskite solar cells. (a) different modulation time at the forward bias of $2 \mathrm{~V}$. (b) Different modulation time at the reversed bias of $-0.5 \mathrm{~V}$.

\section{Conclusions}

In summary, we report that the charge carrier transport can be manipulated by electric field modulation in quasi-2D perovskite solar cells. Using $\mathrm{V}_{\mathrm{app}}<\mathrm{V}_{\mathrm{bi}}$ with proper modulation time, the parameters of photovoltaics can be improved, which indicates that electric polarization formed by electric field modulation enhances charge carrier transport. When the forward bias $V_{a p p}>V_{b i}$ or reversed bias applied, the device performance was reduced. Furthermore, the quenching PL signal indicates that electric field modulation is responsible for charge carrier transport in quasi-2D perovskite solar cells. More importantly, we found that $\Delta \mathrm{PL} / \mathrm{PL}_{0}$ show obvious low $n$ value peak in the worse performance devices with improper electric field modulation. This further confirms that electric field modulation plays a crucial role for carrier transport in quasi-2D perovskites. The electric field modulation functioning as an effective experimental method to form fast charge transfer between quantum wells through electric polarization allows the charge carrier transport from low $n$ 
value nanoplates to high $n$ value nanoplates to develop high-efficiency quasi-2D perovskite solar cells.

Supplementary Materials: The following are available online at https://www.mdpi.com/article/10 .3390/en14217402/s1, Figure S1: Steady state photoluminescence spectra of perovskite films from front sides at different preheating temperatures, Figure S2: Schematic diagram of the CELIV test of perovskite solar cell.

Author Contributions: Conceptualization, X.Z. and Y.X.; methodology, X.Z., Y.X. and L.P.; formal analysis, X.Z. and Y.X.; investigation, X.Z., L.P., J.L. and H.Y.; writing-original draft preparation, X.Z.; writing-review and editing, X.Z. and Y.X.; funding acquisition, X.Z. and Y.X. All authors have read and agreed to the published version of the manuscript.

Funding: This research was funded by the Fundamental Research Funds for the Central Universities (Grant No. S21JB0100020); Project funded by China Postdoctoral Science Foundation (Grant No. 2021M690336); The National Natural Science Foundation of China (Grant No. 62104016); The Central Government Guide Local Funds for Scientific and Technological Development (Grant No. 2020ZYYD032); Research Fund for the Doctoral Program (2020036); and the High-level Cultivation Program (202108404) of Huanggang Normal University.

Institutional Review Board Statement: Not applicable.

Informed Consent Statement: Not applicable.

Data Availability Statement: The data that supports the findings of this study are available within the article (and its Supplementary Materials).

Acknowledgments: We acknowledge financial support provided by the Fundamental Research Funds for the Central Universities (Grant No. S21JB0100020); Project funded by China Postdoctoral Science Foundation (Grant No. 2021M690336); The National Natural Science Foundation of China (Grant No. 62104016); The Central Government Guide Local Funds for Scientific and Technological Development (Grant No. 2020ZYYD032); Research Fund for the Doctoral Program (2020036); and the High-level Cultivation Program (202108404) of Huanggang Normal University.

Conflicts of Interest: The authors declare no conflict of interest.

\section{References}

1. Lee, J.-W.; Dai, Z.; Han, T.-H.; Choi, C.; Chang, S.-Y.; Lee, S.-J.; De Marco, N.; Zhao, H.; Sun, P.; Huang, Y.; et al. 2D perovskite stabilized phase-pure formamidinium perovskite solar cells. Nat. Commun. 2018, 9, 3021. [CrossRef]

2. Ma, C.; Shen, D.; Ng, T.-W.; Lo, M.-F.; Lee, C.-S. 2D Perovskites with short interlayer distance for high-performance solar cell application. Adv. Mater. 2018, 30, e1800710. [CrossRef]

3. Cohen, B.-E.; Wierzbowska, M.; Etgar, L. High efficiency and high open circuit voltage in quasi 2D perovskite based solar cells. Adv. Funct. Mater. 2016, 27, 1604733. [CrossRef]

4. Yang, W.S.; Park, B.-W.; Jung, E.H.; Jeon, N.J.; Kim, Y.C.; Lee, D.U.; Shin, S.S.; Seo, J.; Kim, E.K.; Noh, J.H.; et al. Iodide management in formamidinium-lead-halide-based perovskite layers for efficient solar cells. Science 2017, 356, 1376-1379. [CrossRef]

5. Ren, M.; Cao, S.; Zhao, J.; Zou, B.; Zeng, R. Advances and challenges in two-dimensional organic-inorganic hybrid per-ovskites toward high-performance light-emitting diodes. Nano Micro Lett. 2021, 13, 1-36. [CrossRef] [PubMed]

6. Liu, P.; Yu, S.; Xiao, S. Research progress on two-dimensional (2D) halide organic-inorganic hybrid perovskites. Sustain. Energy Fuels 2021, 5, 3950-3978. [CrossRef]

7. Zhang, L.; Sun, C.; He, T.; Jiang, Y.; Wei, J.; Huang, Y.; Yuan, M. High-performance quasi-2D perovskite light-emitting diodes: From materials to devices. Light Sci. Appl. 2021, 10, 61. [CrossRef] [PubMed]

8. Zhang, X.; Munir, R.; Xu, Z.; Liu, Y.; Tsai, H.; Nie, W.; Li, J.; Niu, T.; Smilgies, D.; Kanatzidis, M.G.; et al. Phase Transition Control for High Performance Ruddlesden-Popper Perovskite Solar Cells. Adv. Mater. 2018, 30, e1707166. [CrossRef] [PubMed]

9. Roghabadi, F.A.; Alidaei, M.; Mousavi, S.M.; Ashjari, T.; Tehrani, A.S.; Ahmadi, V.; Sadrameli, S.M. Stability progress of perovskite solar cells dependent on the crystalline structure: From 3D ABX3 to 2D Ruddlesden-Popper perovskite absorbers. J. Mater. Chem. A 2019, 7, 5898-5933. [CrossRef]

10. Wang, K.; Li, Z.; Zhou, F.; Wang, H.; Bian, H.; Zhang, H.; Wang, Q.; Jin, Z.; Ding, L.; Liu, S. Ruddlesden-Popper 2D Com-ponent to Stabilize $\gamma$-CsPbI3 Perovskite Phase for Stable and Efficient Photovoltaics. Adv. Energy Mater. 2019, 9, 1902529. [CrossRef]

11. Chen, Y.; Sun, Y.; Peng, J.; Tang, J.; Zheng, K.; Liang, Z. 2D Ruddlesden-Popper Perovskites for Optoelectronics. Adv. Mater. 2018, 30, 1703487. [CrossRef] [PubMed] 
12. Zheng, K.; Chen, Y.; Sun, Y.; Chen, J.; Chábera, P.; Schaller, R.; Al-Marri, M.J.; Canton, S.E.; Liang, Z.; Pullerits, T. Inter-phase charge and energy transfer in Ruddlesden-Popper 2D perovskites: Critical role of the spacing cations. J. Mater. Chem. A 2018, 6, 6244-6250. [CrossRef]

13. Tsai, H.; Nie, W.; Blancon, J.-C.; Stoumpos, C.; Asadpour, R.; Harutyunyan, B.; Neukirch, A.J.; Verduzco, R.; Crochet, J.; Tretiak, S.; et al. High-efficiency two-dimensional Ruddlesden-Popper perovskite solar cells. Nat. Cell Biol. 2016, 536, 312-316. [CrossRef]

14. Zhang, J.; Qin, J.; Wang, M.; Bai, Y.; Zou, H.; Keum, J.K.; Tao, R.; Xu, H.; Yu, H.; Haacke, S.; et al. Uniform Permutation of Quasi-2D perovskites by vacuum poling for efficient, high-fill-factor solar cells. Joule 2019, 3, 3061-3071. [CrossRef]

15. Xiao, X.; Dai, J.; Fang, Y.; Zhao, J.; Zheng, X.; Tang, S.; Rudd, P.N.; Zeng, X.C.; Huang, J. Suppressed Ion Migration along the In-Plane Direction in Layered Perovskites. ACS Energy Lett. 2018, 3, 684-688. [CrossRef]

16. Kim, H.; Kim, J.S.; Heo, J.-M.; Pei, M.; Park, I.-H.; Liu, Z.; Yun, H.J.; Park, M.-H.; Jeong, S.-H.; Kim, Y.-H. Proton-transfer-induced 3D/2D hybrid perovskites suppress ion migration and reduce luminance overshoot. Nat. Commun. 2020, 11, 3378. [CrossRef]

17. Lin, Y.; Bai, Y.; Fang, Y.; Wang, Q.; Deng, Y.; Huang, J. Suppressed ion migration in low-dimensional perovskites. ACS Energy Lett. 2017, 2, 1571-1572. [CrossRef]

18. Liu, P.; Wang, W.; Liu, S.; Yang, H.; Shao, Z. Fundamental understanding of photocurrent hysteresis in perovskite solar cells. Adv. Energy Mater. 2019, 9, 1803017. [CrossRef]

19. Cheng, T.; Tumen-Ulzii, G.; Klotz, D.; Watanabe, S.; Matsushima, T.; Adachi, C. Ion migration-induced degradation and efficiency roll-off in quasi-2D perovskite light-emitting diodes. ACS Appl. Mater. Interfaces 2020, 12, 33004-33013. [CrossRef] [PubMed]

20. Liu, F.; Wang, L.; Wang, J.; Wang, F.; Chen, Y.; Zhang, S.; Sun, H.; Liu, J.; Wang, G.; Hu, Y. 2D Ruddlesden-popper perovskite single crystal field-effect transistors. Adv. Funct. Mater. 2021, 31, 2005662. [CrossRef]

21. Li, Z.; Xiao, C.; Yang, Y.; Harvey, S.P.; Kim, D.H.; Christians, J.A.; Yang, M.; Schulz, P.; Nanayakkara, S.U.; Jiang, C.-S.; et al. Extrinsic ion migration in perovskite solar cells. Energy Environ. Sci. 2017, 10, 1234-1242. [CrossRef]

22. Zhang, J.; Chen, R.; Wu, Y.; Shang, M.-H.; Zeng, Z.; Zhu, Y.; Han, L. Extrinsic Movable Ions in MAPbI3 Modulate Energy Band Alignment in Perovskite Solar Cells. Adv. Energy Mater. 2017, 8, 1701981. [CrossRef]

23. Leijtens, T.; Kandada, A.R.S.; Eperon, G.E.; Grancini, G.; D’Innocenzo, V.; Ball, J.M.; Stranks, S.D.; Snaith, H.J.; Petrozza, A. Modulating the electron-Hole interaction in a hybrid lead halide perovskite with an electric field. J. Am. Chem. Soc. 2015, 137, 15451-15459. [CrossRef]

24. Hu, X.; Wang, X.; Fan, P.; Li, Y.; Zhang, X.; Liu, Q.; Zheng, W.; Xu, G.; Wang, X.; Zhu, X.; et al. Visualizing Carrier Transport in Metal Halide Perovskite Nanoplates via Electric Field Modulated Photoluminescence Imaging. Nano Lett. 2018, 18, 3024-3031. [CrossRef] [PubMed]

25. Shi, J.; Xu, X.; Zhang, H.; Luo, Y.; Li, D.; Meng, Q. Intrinsic slow charge response in the perovskite solar cells: Electron and ion transport. Appl. Phys. Lett. 2015, 107, 163901. [CrossRef]

26. Zhang, J.; Zhu, X.; Wang, M.; Hu, B. Establishing charge-transfer excitons in 2D perovskite heterostructures. Nat. Commun. 2020, 11, 2618. [CrossRef] [PubMed]

27. Wu, G.; Li, X.; Zhou, J.; Zhang, J.; Zhang, X.; Leng, X.; Wang, P.; Chen, M.; Zhang, D.; Zhao, K.; et al. Fine Multi-Phase Alignments in 2D Perovskite Solar Cells with Efficiency over 17\% via Slow Post-Annealing. Adv. Mater. 2019, 31, e1903889. [CrossRef] [PubMed]

28. Eames, C.; Frost, J.M.; Barnes, P.; O’Regan, B.C.; Walsh, A.; Islam, M.S. Ionic transport in hybrid lead iodide perovskite solar cells. Nat. Commun. 2015, 6, 7497. [CrossRef]

29. De Bastiani, M.; Dell'Erba, G.; Gandini, M.; D’Innocenzo, V.; Neutzner, S.; Kandada, A.R.S.; Grancini, G.; Binda, M.; Prato, M.; Ball, J.M. Ion migration and the role of preconditioning cycles in the stabilization of the $\mathrm{J}-\mathrm{V}$ characteristics of inverted hybrid perovskite solar cells. Adv. Energy Mater. 2016, 6, 1501453. [CrossRef]

30. Hoke, E.T.; Slotcavage, D.J.; Dohner, E.R.; Bowring, A.R.; Karunadasa, H.I.; McGehee, M.D. Reversible photo-induced trap formation in mixed-halide hybrid perovskites for photovoltaics. Chem. Sci. 2015, 6, 613-617. [CrossRef]

31. Fabregat-Santiago, F.; Garcia-Belmonte, G.; Mora-Seró, I.; Bisquert, J. Characterization of nanostructured hybrid and organic solar cells by impedance spectroscopy. Phys. Chem. Chem. Phys. 2011, 13, 9083-9118. [CrossRef]

32. Zhu, X.; Wang, K.; Zhao, F.; Han, C.; Yang, Q.; Yu, H.; Zhang, F.; Hu, B. Revisiting the Impact of Interfacial Transport Layers on Organic Bulk Heterojunction Systems. ACS Appl. Energy Mater. 2018, 1, 3457-3468. [CrossRef]

33. Lin, Y.; Fang, Y.; Zhao, J.; Shao, Y.; Stuard, S.J.; Nahid, M.M.; Ade, H.; Wang, Q.; Shield, J.E.; Zhou, N. Unveiling the operation mechanism of layered perovskite solar cells. Nat. Commun. 2019, 10, 1008. [CrossRef] [PubMed]

34. Zhu, X.; Xu, H.; Liu, Y.; Zhang, J.; Wang, M.; Ivanov, I.N.; Ovchinnikova, O.S.; Hu, B. Two-Photon Up-conversion photoluminescence realized through spatially extended gap states in Quasi-2D perovskite films. Adv. Mater. 2019, 31, 1901240. [CrossRef] [PubMed] 